\title{
SUSTENTABILIDAD DEAGROECOSISTEMAS
}

\author{
SUSTAINABILITYOF AGROECOSYSTEMS \\ Toro-Mújica, P. ${ }^{* 1}$, García, A. ${ }^{2}$, Gómez-Castro, A.G. ${ }^{2}$, Acero, R. ${ }^{3}$, Perea, J. ${ }^{2}$ \\ y Rodríguez-Estévez, ${ }^{2}{ }^{2}$
}

\begin{abstract}
${ }^{1}$ Pontificia Universidad Católica de Chile. Facultad de Agronomía e Ingeniería Forestal. Av. Vicuña Mackenna 4860. Santiago. Chile. *pmtoro@uc.cl

${ }^{2}$ Departamento de Producción Animal. Facultad de Veterinaria. Universidad de Córdoba. Campus de Rabanales. 14071 Córdoba. España.

${ }^{3}$ Departamento de Organización de Empresas. Universidad de Córdoba. Campus de Rabanales. 14071 Córdoba. España.
\end{abstract}

\section{Palabras clave adicionales}

Dimensiones. Desarrollo sostenible. Versiones de la sustentabilidad.

\section{RESUMEN}

En este trabajo se revisan, desde la perspectiva de los agroecosistemas, los conceptos de sustentabilidad reflejados en sus dimensiones: ecológica, social y económica, así como lo relativo al grado de sustitución entre capital natural y humano que se manifiesta en las versiones de la sustentabilidad: débil, que asume la capacidad de sustitución total entre estos recursos, y fuerte que postula que los sistemas naturales son de mayor entidad que los criterios de eficiencia económica. Entre las características principales de sustentabilidad de los agroecosistemas, la resiliencia, asociada a la estabilidad económica y la estabilidad social, debe ser considerada como la desencadenante de la continuidad o discontinuidad de los sistemas. La conveniencia debe ser abordada abandonando los conceptos antropocéntricos y productivistas del corto plazo en beneficio de una concepción holística de los sistemas de producción. Por último, la escala, espacial o temporal, es el factor que permite una cierta adaptación al sistema analizado, modulando la escala temporal a la jerarquización y complejidad de los sistemas agropecuarios y estableciendo como unidad espacial la explotación, sin olvidar la interacción con las actividades de explotaciones adyacentes.

\section{SUMMARY}

In this paper we review, from the perspective of

\begin{abstract}
AdDiTIONAL KEYWORDS
Dimensions. Sustainable development. Sustentability versions.
\end{abstract}

agroecosystems, the concept of sustainability as reflected in its ecological, social and economic dimensions. This work also focuses on the degree of substitution between natural and human capitals, manifested in the two versions of sustainability: weak sustainability, which assumes complete substitutability between these resources, and strong sustainability which postulates that natural systems are of greater importance than economic efficiency criteria. Among the main features of agro-ecosystem sustainability, resilience, associated to economic and social stabilities, should be regarded as the trigger for the continuity or discontinuity of the systems. Convenience should considered, after abandoning the anthropocentric, and short term, perspective, by developing a holistic consideration of production systems. Finally, the space and time scales, are the factor that allows a certain adaptation to the analyzed system, by modulating the timescale to hierarchy and complexity of agricultural systems, and after considering farm as the spatial unit, without forget its interaction with adjacent farm activities.

\section{INTRODUCCIÓN}

Como señala el informe Brundtland Nuestro futuro común (Comisión Mundial del Medio Ambiente y del Desarrollo, 1988): En el pasado nos preocupábamos por los 
impactos del crecimiento económico en el medio ambiente. Ahora, nos vemos obligados a preocuparnos por los impactos que la presión ecológica, la degradación de los suelos, los regímenes de aguas, la atmósfera y los bosques ejercen sobre nuestras perspectivas económicas. En el pasado más reciente nos vimos forzados a enfrentarnos a un agudo incremento de la interdependencia económica entre las naciones. Ahora debemos acostumbrarnos también a una acelerada interdependencia ecológica. La ecología y la economía están entrelazándose cada vez más tanto local como regional, nacional y mundialmente, en una red de causas y efectos. En otras palabras, la protección del medio ambiente no puede lograrse sin que la política económica se complemente con una perspectiva medioambiental, y a su vez, dado que la protección de los recursos naturales se relaciona directamente con su nivel de demanda por parte de la sociedad, no se debe ignorar la dimensión social de la sustentabilidad.

Así, la percepción del concepto de sustentabilidad ha sufrido un proceso de transformación, pasando de una visión exclusiva del deterioro medioambiental a otra más global, en la que también son trascendentes otros aspectos, como los vinculados con la calidad de vida del ser humano. Esta nueva visión demanda la vinculación del desarrollo al medioambiente, lo que conlleva a un enfoque más integrador de la ecología y economía. La necesidad de este proceso de transformación se ha evidenciado por medio de variados fenómenos, destacándose aquellos relacionados con el cambio climático, cuyo principal origen ha sido el mal uso de los recursos por parte del hombre, consecuencia del cambio social global debido al aumento de la población, el crecimiento económico, el avance tecnológico y la pobreza (Jiménez-Herrero, 2001).

La diferente capacidad de sustitución que los economistas y ecólogos plantean entre capital humano y capital natural han llevado a la existencia de dos versiones de sustentabilidad: una sustentabilidad débil que postula la posibilidad de la total sustitución del recurso capital natural por el de capital humano, y por lo tanto la eventualidad de degradar al primero si es posible su recuperación a través de la tecnología (Mas-Colell, 1994); y una sustentabilidad fuerte, para la que el capital natural no es sustituible por ningún tipo de capital humano (Daly, 1992).

En los sistemas ganaderos la versión de sustentabilidad se empezó a desarrollar en la segunda mitad de la década de los 90 del siglo XX (Speeding, 1995; Heitschmidt et al., 1996 y Vavra, 1996); habiendo sufrido un proceso de cambio en los últimos años. Desde sistemas, como es el caso de la ganadería intensiva o especializada, en los que prevalece la versión de sustentabilidad débil, y predominan objetivos meramente productivistas, fácilmente valorables a través de los rendimientos y la viabilidad económica (Giorgis, 2009); se ha pasado, en la actualidad a potenciar la versión fuerte de sustentabilidad, que pretende el equilibrio de los elementos sociales, económicos y ambientales; como sucede en la ganadería extensiva (Nahed et $a l ., 2006)$ o en la ganadería ecológica (que está más regulada) en la que se potencia el uso de razas autóctonas, sistemas tradicionales de producción y la utilización de recursos locales (Frías, 1998; Fernández, 2005).

Como parte especialmente relevante del panorama general hay que indicar que los agroecosistemas ganaderos y la sociedad están unidos en una extensa y compleja simbiosis, expresada en relaciones de mutua dependencia, dado que los agroecosistemas son la base de un sinnúmero de estructuras productivas. Por otro lado, y considerando la capacidad de intervención humana, la salud de los agroecosistemas depende principalmente de los usos y cuidados que se pongan en juego. En los sis- 


\section{SUSTENTABILIDAD DEAGROECOSISTEMAS}

temas agroganaderos, las relaciones de dependencia entre las dimensiones de sustentabilidad son básicas para la continuidad de las explotaciones. A modo de ejemplo, en la ganadería de pequeños rumiantes en los países de Sur y el Este del Mediterráneo la necesidad de incrementar la baja productividad de los sistemas locales de producción ha llevado al incremento de las poblaciones de pequeños rumiantes, lo que trae consigo consecuencias medioambientales, derivadas del sobrepastoreo y de la consiguiente degradación de suelos y deforestación. En el otro extremo, encontramos a los países de la Unión Europea, donde la pérdida de rentabilidad de las explotaciones, así como el envejecimiento de los ganaderos y las dificultades para su relevo generacional, han provocado la reducción en el número de cabezas y rebaños, situación que podría tener serias consecuencias sociales y medioambientales, puesto que los ovinos y caprinos contribuyen a la conservación de áreas marginales y al mantenimiento de los medios de subsistencia del entorno rural (Castel et al., 2003).

En la presente revisión se plantea como objetivo un análisis de la sustentabilidad, en un contexto renovado, con especial atención a los agroecosistemas ganaderos, en lo relativo a las características y propiedades que la condicionan.

\section{DEFINICIÓNDE SUSTENTABILIDAD}

La sustentabilidad es un concepto antiguo; su origen se encuentra en la palabra Nachhaltigkeit del jurista alemán Hans Carlowitz (1645-1714), quien desarrolló en 1713 una teoría sobre la utilización óptima de los bosques, que fueron las fuentes de energía para la protoindustria de hierro y plata. Carlowitz planteó que el volumen de producción de la industria no podía ser superior a la velocidad de reproducción de los bosques (Marquardt, 2006).

Su raíz latina proviene de sustinere que significa sostener, mantener, sustentar, aunque la influencia del vocablo inglés sustainable añade a estos significados otros como soportar y tolerar, de ahí que se haya impuesto el epíteto de sostenible, en lugar de sustentable. La introducción del término, sin embargo, no fue hasta 1974 en la declaración de Cocoyot, con motivo de una reunión celebrada por Naciones Unidas en México y fue asumido en 1980 en la publicación de la Estrategia Mundial de la Conservación de la UICN (Unión Internacional de la Conservación de la Naturaleza), en 1980 (Luffiego y Rabadán, 2000).

Hoy en día existen diversas opiniones sobre el significado de sustentabilidad, siendo la más aceptada la propuesta por la Comisión Mundial para el Medio Ambiente y el Desarrollo (1988), que la define como la relación entre los sistemas humano y ecológico que permite mejorar y desarrollar la calidad de vida, manteniendo al mismo tiempo, la estructura, las funciones y la diversidad de los sistemas que sustentan la vida.

Otra definición es la señalada por Mayer (2008): el nivel de consumo y actividad humana que puede continuar dentro de un futuro previsible, a fin de que los sistemas que proporcionan bienes y servicios a los seres humanos persistan indefinidamente. Las implicaciones prácticas de esta definición son diversas, yendo desde el consumo de recursos con respeto a su tasa de renovación, a la eficiencia de la utilización de los recursos, y a la equidad de su uso a través de las sociedades y generaciones, con diferentes énfasis de acuerdo a la disciplina y la ideología política (Ulgiati y Brown, 1998; Parris y Kates, 2003).

El concepto de sustentabilidad no ha estado libre de polémica y discusión debido a su ambigüedad y generalidad, que permite que cualquier política pueda tener como objetivo el Desarrollo Sostenible (Labandeira, 2007). 
La utilización y aprobación del concepto sustentabilidad, implica el haber comprendido que el mundo no es tan ancho e ilimitado como se creía, lo que queda expresado claramente en un trozo del texto de Victoria Chitepo, Ministra de Recursos Naturales y Turismo de Zimbabwe, en Nuestro futuro común Se creía que el cielo es tan inmenso y claro que nada podría cambiar su color, nuestros ríos tan grandes y sus aguas tan caudalosas que ninguna actividad humana podría cambiar su calidad, y que había tal abundancia de árboles y de bosques naturales que nunca terminaríamos con ellos (Macedo, 2005). De este modo, la sustentabilidad global resulta imposible a menos que la población humana y el nivel de demanda de recursos, como máximo, se equiparen con la capacidad de carga de la tierra, lo que sin embargo, no significa que cada país, región o zona, requieran comportarse homogéneamente con respecto a todos los recursos y al principio de capacidad de carga o sustentación; dado que lo que a una región le falta en su proceso de desarrollo a otra le sobra y deberá haber complejas compensaciones e incluso ritmos muy diferentes en la incorporación o adaptación de las distintas áreas (Allende, 2000).

Además, debe asumirse el hecho, tal como lo señala la tradición africana, de que: el hombre no es el dueño del universo, sino más bien el núcleo, pero en el sentido de ser el amigo, el beneficiario y el usuario, debiendo vivir en armonía con él, obedeciendo las leyes de orden natural, moral y místico (Mbiti, 1996, citado por Mebratu, 1998); pues, si este orden es perturbado indebidamente, será el hombre quién sufra la mayoría de las consecuencias. La lección más importante que se puede extraer de las tradiciones y creencias indígenas es la visión holística, que es inherente a todas las creencias, además de la importancia que se atribuye a la comunicación constante con la naturaleza. Si bien es cierto que, y a pesar de la brillantez de las enseñanzas de las tradiciones africanas, actualmente vivimos en un mundo muy complejo, que abarca un ámbito que va más allá de estas enseñanzas. Sin embargo, la sabiduría tradicional tiene mucho que ofrecer en términos de vivir en armonía con la naturaleza y en sociedad, siendo este uno de los principios fundamentales del concepto sustetabilidad (Mebratu, 1998).

Dentro de este marco, los sistemas agropecuarios no podían ser menos, y así sus objetivos se han ampliado como indican Valerio et al. (2009) y Giorgis (2009), al señalar la dualidad de los fines de la empresa pecuaria; que por una parte busca la rentabilidad económica y social (fijando la población al medio rural en condiciones dignas) y por otra la preservación del patrimonio ganadero y ambiental recibido.

\section{Agricultura sostenible}

La agricultura sostenible se refiere a un modo de agricultura que intenta proporcionar un rendimiento sostenido a largo plazo, a través la gestión de tecnologías ecológicamente racionales. Siendo necesaria que la agricultura sea considerada como un ecosistema (agroecosistema) y, al mismo tiempo, que la agricultura y la investigación no se enfoquen en la obtención de altos rendimientos de un producto en particular, sino a la optimización del sistema en su conjunto (Altieri, 1992a). Esta concepción ecológica es formulada por el IFOAM (2005) como el principio de Ecología, que postula que la agricultura orgánica debe estar basada en sistemas y ciclos ecológicos vivos, trabajar con ellos, emularlos y ayudar a sostenerlos.

Según Marten (1988) un agroecosistema es un complejo integrado por aire, agua, suelo, plantas, animales, microorganismos, y todo lo demás, en una zona delimitada que ha sido modificado por personas a los efectos de la producción agríco- 


\section{SUSTENTABILIDAD DEAGROECOSISTEMAS}

la y ganadera, señalando que un agroecosistema puede ser de cualquier tamaño, por ejemplo: un solo campo, una granja, el paisaje agrícola de un pueblo, una región o una nación pero, en definitiva, como recoge Okey (1996) el agroecosistema es un conjunto de ecosistemas especializados, que son controlados para la generación de productos agropecuarios.

La preocupación por el constante aumento de la degradación de los recursos naturales pertenecientes a los agroecosistemas ha llevado al concepto de agricultura sostenible; que puede ser definida como la que a largo plazo mejora la calidad del medio ambiente $y$ de los recursos base de los que depende, ofrece alimento y fibra para satisfacer las necesidades humanas básicas, es económicamente viable y mejora la calidad de vida de agricultores y de la sociedad en su conjunto (Weil, 1990).

La generalidad de la expresión agricultura sostenible la ha dotado de popularidad, ya que atañe no sólo a las personas interesadas en el medio ambiente y en la agricultura saludable, sino también a los interesados en las consecuencias de las dimensiones económicas y sociales de la producción agropecuaria (Schaller, 1993).

De este modo, los principios claves para potenciar la sustentabilidad de agroecosistemas, basados en la conservación de las energías renovables, la adaptación del cultivo al medio ambiente y el mantenimiento de un nivel moderado, pero sostenible de productividad, han de enfocarse al desarrollo de tecnologías y prácticas que, tal como señala Pretty (2008):

i) Integren procesos biológicos y ecológicos, como los ciclos de los nutrientes, la fijación de nitrógeno, la regeneración del suelo, la alelopatía, la competencia, la depredación y el parasitismo en los procesos de producción de alimentos.

ii) Reduzcan al mínimo el uso de insumos no renovables o que causen daño al medio ambiente o a la salud de agricultores, ganaderos y consumidores

iii) Hagan un uso productivo de los conocimientos y aptitudes de los agricultores y ganaderos, mejorando así su autosuficiencia y sustituyendo por capital humano costosos insumos externos

iv) Hagan un uso productivo de la capacidad colectiva de las personas de trabajar juntos para resolver problemas agroganaderos y de los recursos naturales, tales como plagas, enfermedades, explotación de cuencas hidrográficas, el riego, aprovechamiento de los bosques, la gestión de crédito, etc.

Los puntos anteriores, sin embargo, no implican descartar cualquier tecnología o práctica por motivos ideológicos, dado que si se trabaja en una tecnología que mejore la productividad de los agricultores y no sea excesivamente dañina para el medio ambiente, es probable que tenga beneficios para la sustentabilidad global del sistema (Pretty, 2008). Sin embargo, la compleja naturaleza de las interacciones entre producción agraria y medioambiente natural hace difícil conocer qué métodos y sistemas de producción puedan conducir a la sustentabilidad en diferentes localizaciones (Youngberg y Harwood, 1989). Por lo tanto, la identificación de tecnologías como sustentables es cuestionable, dado que éstas se basan en hipótesis de manejo sostenible de recursos naturales para mantener su capacidad productiva a través del tiempo, lo que requiere un proceso de monitoreo y reevaluación constante (Meul et al., 2008, Toro et al., 2010). La agroecología, cuyo propósito de estudiar, diagnosticar y proponer alternativas para la utilización de cantidades bajas de insumos en agroecosistemas, se traslapa con el concepto de agricultura sostenible. Sin embargo, tal disciplina presenta una falencia al responsabilizar al ámbito tecnológico de la falta de sustentabilidad de los agroecosistemas (Altieri, 1989). En consecuen- 
cia la sustentabilidad no puede ser asociada a un set de prácticas o métodos agrarios, dado que la capacidad de una cierta tecnología para comportarse como sustentable depende generalmente de las peculiaridades del contexto en el que se utiliza (Rigby y Cáceres, 2001).

En la producción ganadera, la sustentabilidad se entiende como la producción de una cantidad constante de productos animales (carne, leche, fibra) en una determinada base de tierra en forma indefinida (Masera et al., 1999). Es decir, que la extracción de los productos no hace decaer la capacidad de la tierra para seguir proporcionando los materiales (por ejemplo, forraje) para una nueva extracción. Sin embargo, aunque el potencial de producción de productos básicos de la tierra está protegido por este enfoque, la integridad del ecosistema, es decir, la interacción de las plantas y animales autóctonos con su medio ambiente, no es considerada (Vavra, 1996).

En este ámbito, las políticas agrarias en los países desarrollados se comprometen cada día más con la obtención de una actividad agroganadera sostenible, enfatizando la producción sana, con métodos que garanticen la biodiversidad y los productos de alta calidad. Así desde la visión original de la PAC (I) centrada en el aumento de la productividad, que obviaba las interacciones entre plantas y animales autóctonos con su medio ambiente, potenciando el desequilibrio entre agricultura y medioambiente, al favorecer el uso de fertilizantes, la especialización (ganadería o agricultura), o el incremento de explotaciones de mediano o gran tamaño, se pasa a la PAC II que subsana dichos inconvenientes al incorporar una Política Agroambiental Comunitaria (a través de la aprobación del Reglamento CEE 2078/92 sobre ayudas para fomentar métodos de producción agraria compatibles con las exigencias de la protección del medio ambiente y conservación del espacio natural) que contempla reformas que entre otros aspectos implican (Morán, 2000):

- Reducción sustancial del uso de fertilizantes y productos fitosanitarios, e introducción de la agricultura y ganadería ecológica.

- Extensificación de las producciones y transformación de tierras de cultivo en pastos.

- Reducción de la carga ganadera en los terrenos de pastos.

- Utilización de prácticas agronómicas compatibles con la protección del medio ambiente y de los recursos naturales.

- Mantenimiento de tierras agrarias y forestales abandonadas.

- Retirada de tierras de cultivo, por un período de 20 años, para uso ambiental (establecimiento de parques naturales, protección de sistemas hidrológicos, etc.).

- Gestión de espacios para uso recreativo.

- Medidas formativas para mejorar la cualificación de los agricultores y ganaderos en relación con la aplicación de las medidas agroambientales.

\section{SuSTENTABILIDAD DE AGROECOSISTEMAS}

La sustentabilidad de los agroecosistemas se determina a través de tres características principales, la resiliencia frente a las perturbaciones, tanto naturales como antropogénicas (por ejemplo, la capacidad de mantener la producción a pesar de ciertos cambios climáticos, la resistencia de las dehesas al continuo y a veces abusivo, aprovechamiento ganadero...); la conveniencia para las sociedades humanas (generación de productos como fuente de alimento o con especial marchamo de calidad); y la escala tanto a nivel temporal como espacial (Mayer, 2008).

La resiliencia se define como la capacidad de un sistema para tolerar perturbaciones de cierta magnitud antes de trasladarse a una región diferente del espacio de estado controlada por un conjunto distinto de procesos (Carperter et al., 2001), en otras 


\section{SUSTENTABILIDAD DEAGROECOSISTEMAS}

palabras, antes de transformarse en otro sistema gobernado por un conjunto diferente de controles. Gunderson y Holling (2001) definen el término resiliencia como la capacidad de un sistema para someterse a una perturbación y mantener sus funciones y controles. El concepto y la medición de la resiliencia, tal como se han desarrollado en la ecología, se inspiró en sistemas dinámicos y en la teoría de la catástrofe (Fiksel, 2006). La resiliencia de un modelo de producción (o régimen) es el grado en que el sistema puede adaptarse a las perturbaciones sin pasar a un nuevo régimen (Grimm y Wissel, 1997; Carpenter et al., 2001). El sistema permanece en ese régimen a causa de reacciones entre sus componentes que impiden que se aleje demasiado de su estado inicial. Sin embargo, si un sistema avanza hacia un nuevo régimen, los nuevos feed- back se adecuaran para mantener el sistema en este nuevo estado (Mayer, 2008). Es decir, cuando un ecosistema se altera con el fin de convertirlo en un agroecosistema, el equilibrio original y la resiliencia se modifican, siendo reemplazado por otra combinación de factores y restricciones ecológicas y socioeconómicas (Gliessman, 1995). Una situación común ocurre cuando en los sistemas pecuarios, por ejemplo en el aprovechamiento de las dehesas, se intensifica la producción a través del aumento de la carga animal, la mayor demanda de nutrientes puede ser satisfecha por el sistema hasta un cierto límite, en el que se hace necesaria la incorporación de energía externa a través del uso de concentrados u otros alimentos, si no se aportan estas medidas paliativas, el sistema evolucionará a una nueva situación, disclimácica, en la que perderá uno de sus estratos botánicos fundamentales (la arboleda) y también el estrato herbáceo. Si la evolución al nuevo régimen, se produce por una disminución de la carga ganadera, una vez que se traspasa cierto límite, la regresión a la vegetación climax, el bosque mediterráneo, determinará una nueva situación cuya sostenibilidad depende de otros parámetros en nada relacionados con los de la dehesa originaria (Garcia et al., 2009). En el caso particular de los agroecosistemas, la resiliencia se relaciona con los servicios naturales que proveen los ecosistemas (como circulación de nutrientes, oferta de agua, catálogo de enemigos naturales como plagas y enfermedades), lo que no sólo depende de la cuantía neta total de energía para atender a la productividad primaria (determinada por el flujo de energía) sino además de la energía que se mantiene en el sistema para preservar especies nativas y naturalizadas después de la cosecha (Halberg, 1999). Cuando estos servicios se reducen, o se hacen inexistentes en el sistema original o, en definitiva, son inferiores o notablemente diferentes, a lo demandado por las indicadas formas energéticas, es necesario realizar un aporte de energía al sistema, ya sea en forma de fertilizantes, plaguicidas, herbicidas o mejora de genotipos (van Ittersum y Rabbinge, 1997). La resiliencia, se asocia con la estabilidad de los agroecosistemas, que puede ser medida como la oscilación en torno al promedio de cada actividad, de forma que agroecosistemas con mayor amplitud en sus oscilaciones serán menos resilientes (Viglizzo, 1986), así, la resiliencia es definida como la capacidad de un sistema para alcanzar y mantener de forma estable su dinámica de equilibrio, preservando las ganancias del sistema en el tiempo, estipulando que todos los otros factores y condiciones siguen siendo normales en virtud de un promedio (Nahed et al, 2006). Sin embargo, se tiende a asociar resiliencia con el comportamiento originado a raíz de un cambio en el equilibrio, mientras que la estabilidad se refiere más a la capacidad de resistir dichos cambios (Okey, 1996). Marten (1988) muestra la relación entre sustentabilidad y estabilidad mediante esquemas que representan la producción en relación a un eje temporal (figura 1). 
Los agroecosistemas, como los sistemas de producción ecológicos, con esquemas productivos más articulados, es decir con actividades conectadas a través de secuencias de uso del mismo espacio (en los que la rotación de cultivos se completa con animales que además pueden ejercer acciones complementarias entre sí) son menos productivos pero más estables que los menos articulados (Viglizzo y Roberto, 1998). Del mismo modo, la diversificación actúa como un factor anti-riesgo al distribuir el riesgo ambiental, tanto físico como económico, entre distintas actividades que difieren en su estacionalidad y distribución espacial. Así, ciertos disturbios circunstanciales pueden afectar severamente a una determinada actividad agropecuaria en un momento dado, pero es improbable que perturben por igual a todas las actividades que integran el ciclo productivo del sistema
(Fernández, 2005). Esto se puede asociar con la diversidad en ecosistemas naturales, donde ecosistemas maduros que no han sido alterados, poseen una mayor biomasa, biodiversidad y heterogeneidad espacial (estratos de vegetación), siendo ricos en relaciones tróficas complejas y presentando un alto grado de organización, características que tienen fuertes implicaciones en el control de plagas y malezas y en la descomposición de la materia orgánica (Edwards et al., 1993).

La conveniencia hace referencia a la aptitud de un determinado régimen para los seres humanos (Troell et al., 2005) o sus sistemas de producción. Así, una tierra desprovista de cualquier forma de vida, salvo las bacterias, podría tener muy alta resiliencia, pero una muy baja conveniencia. La introducción de especies vegetales o animales, especialmente las domésticas,

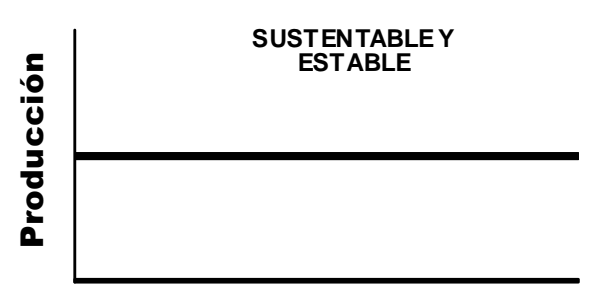

Tiempo

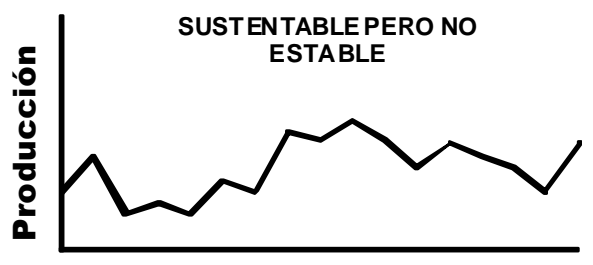

Tiempo

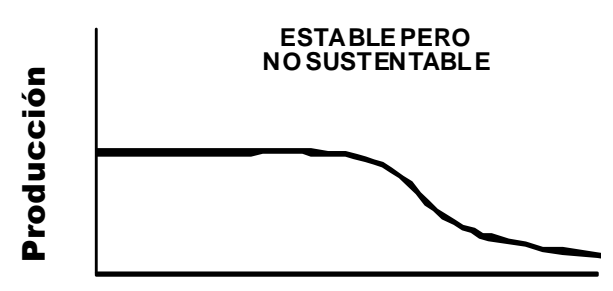

Tiempo

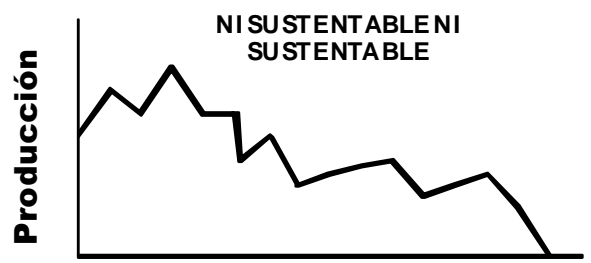

Tiempo

Adaptado de Marten (1998).

Figura 1. El significado de estabilidad y sustentabilidad en términos de la producción en el curso del tiempo. (The meaning of stability and sustainability in terms of production over time). 


\section{SUSTENTABILIDAD DEAGROECOSISTEMAS}

aumenta la conveniencia del sistema aún a riesgo de comprometer y probablemente perjudicar su resiliencia. De manera que, las características deseables de los regímenes involucran decisiones subjetivas, tanto para el valor óptimo de las características. Por ejemplo, ¿qué nivel de riesgo de enfermedad es tolerable en una sociedad? (Mayer, 2008). La inequidad existente entre las economías de distintos países, potencia aún más el grado de subjetividad de este concepto, dada la diferente prioridad que se le otorga a las necesidades humanas (básicas, educación, empleo, ocio) dependiendo del nivel socio-económico. Ejemplos en agroecosistemas pueden encontrarse al observar las distintas posiciones sobre el nivel de aprovechamiento de los recursos naturales en los parques nacionales españoles (Campos y Carrera, 2007), en las decisiones para el control de plagas o enfermedades que afecten a la producción vegetal o pecuaria, en el nivel al que podría llevarse el suministro de una alimentación equilibrada para los animales, o al evaluar las distintas rentabilidades e impactos medioambientales que se generan a partir de la gama de modelos de producción existentes entre ganadería intensiva y ecológica.

Al hablar de sustentabilidad, se hace dentro de un orden, de una escala, es decir, dentro de un período de tiempo lo suficientemente largo como para que sostenerse equivalga a durar aceptablemente y lo bastante corto como para no perderse en disquisiciones (Folch, 1998). Algunos sostienen que la sustentabilidad significa mantener siempre; pero nada dura para siempre, ni siquiera el universo en su conjunto. Sustentabilidad por lo tanto, no puede significar una vida infinita o no sería sostenible (dada la imposibilidad de evolución y adaptación al cambio en las condiciones medioambientales). En lugar de ello, el concepto sostenible debe hacer mención a una vida que sea coherente con el sistema y escalas de tiempo y espacio en que vivimos (Costanza y Patten, 1995).
En la figura 2 se indica la relación entre la vida y una escala de tiempo y espacio, por medio del trazado de la curva de un hipotético sistema de esperanza de vida en el eje y contra la escala de tiempo y espacio en el eje de las x. Se observa que a medida que se avanza en complejidad en el eje de las $\mathrm{x}$ aumenta la esperanza de vida, sin embargo, ningún sistema se espera que tenga una vida infinita. Un sistema sostenible en este contexto es, pues, aquel que alcanza su esperanza de vida plena en la jerarquía anidada de los sistemas dentro de la cual se inserta. En tanto que un sistema susceptible o frágil es aquel que sobrepasa su esperanza de vida, y por lo tanto no posee la capacidad de adaptarse con suficiente rapidez.

Este entramado jerárquico de sistemas y subsistemas en un rango de escalas de tiempo y espacio es conocido como Metasistema. Los seres humanos serán sostenibles en este metasistema si logran su duración de vida normal máxima. Pensado como un experimento, ¿qué sucedería a la sustentabilidad si todos los seres humanos individuales realmente vivieran por siempre? La inmortalidad de cualquier subsistema no es sostenible, ya que corta la adaptación evolutiva. La clave es la diferenciación entre los cambios debidos a la normalidad de vida y los cambios que acortan la vida útil del sistema. En virtud de esta definición, cualquier factor que reduce la longevidad del sistema natural también reduce su sustentabilidad. Por lo tanto, factores como los incendios, las epizootias, los accidentes, y un sinnúmero de otras causas disminuyen la sustentabilidad (Costanza y Patten, 1995).

En el ámbito de la producción animal, si se considera por ejemplo un sistema de producción mixto carne-leche en la zona centro sur de Chile, existen distintas esperanzas de vida para cada subsistema, es así como dentro de la producción de forraje se puede mencionar que la duración media de una pradera artificial de alfalfa es de cuatro 
a seis años, la vida media de las reproductoras de seis a ocho años y de los novillos de 18 a 24 meses. Si aumenta la vida media por ejemplo de los novillos (edad de venta), la disponibilidad de pasto de la pradera disminuirá, provocando su degradación y por lo tanto impidiendo que alcance su esperanza de vida. De este modo, el aumento de la esperanza de vida de cualquiera de los subsistemas dentro de este sistema productivo altera su grado global de sustentabilidad. (Catrileo et al., 2009; Toro et al., 2009).

En la figura 2 se observa que aquellos sistemas que sobrepasan su esperanza de vida son considerados como sistemas susceptibles o frágiles, ya que no poseen la capacidad de adaptarse con suficiente rapidez. Por otra parte, los sistemas con una vida inferior a la esperada son considerados no sostenibles, dado que la longevidad de
En la sustentabilidad de los sistemas agrícolas la escala se asocia a la persistencia o durabilidad de los sistemas, que corresponde su capacidad para mantenerse durante largos períodos (Pretty, 2008). En agroecosistemas la esperanza de vida está directamente asociada a su resiliencia. Dado que toda intervención humana implica una perturbación, la incertidumbre existente en la respuesta del sistema natural ante una modificación antropológica dificulta el conocimiento de su expectativa de vida y con ello de su grado de sustentabilidad. Altieri (1992b) señala variados ejemplos de los efectos que un uso no sustentable de recursos ha ocasionado en agroecosistemas latinoamericanos, proponiendo posibles soluciones. Por otro lado Gliessman (1998) señala que tecnología como cultivos de cobertura, abonos verdes cultivos intercalados, agrosilvicultura y $\mathrm{l}_{\text {i }}$

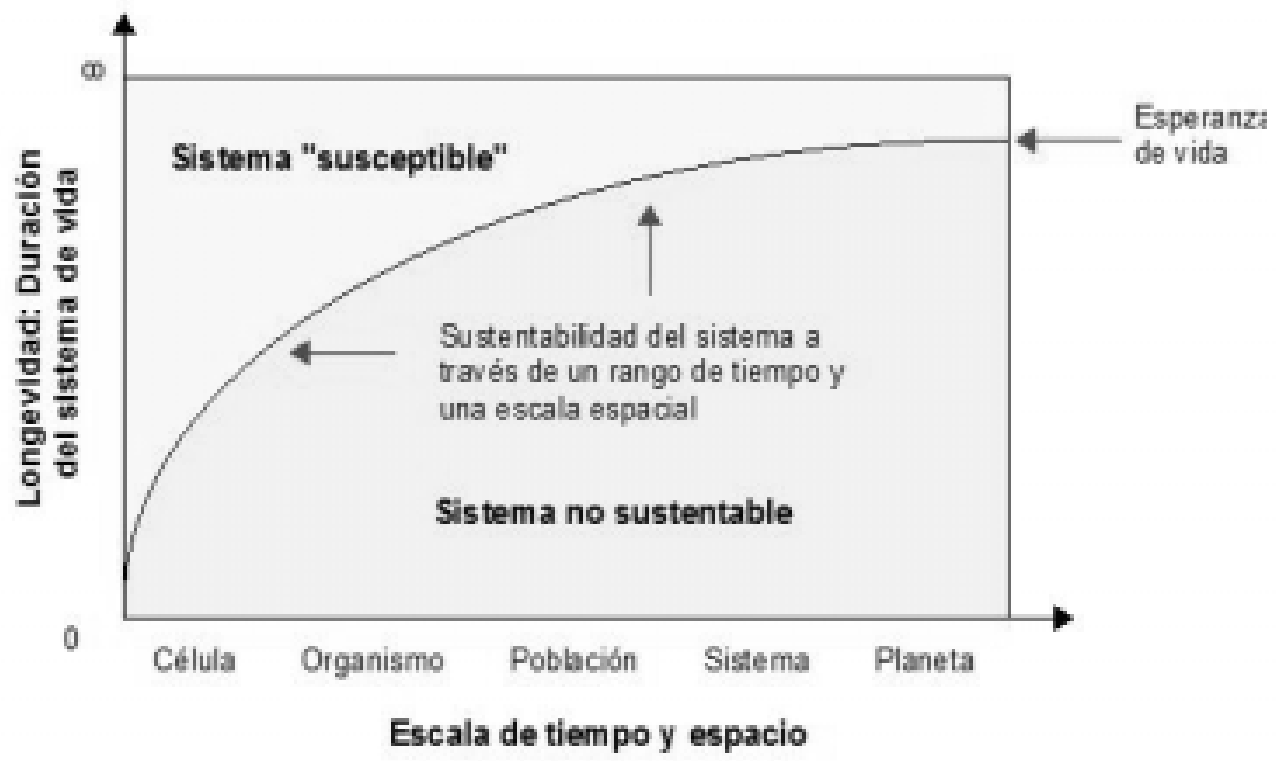

Fuente: Adaptado de Constanza y Patten (1995).

Figura 2. Sustentabilidad como escala (tiempo y espacio) concepto dependiente. (Sustainability as scale (time and space) dependent concept). 


\section{SUSTENTABILIDAD DEAGROECOSISTEMAS}

mezcla de cultivos y ganadería tienden a mejorar la biodiversidad y con ello el funcionamiento de los agroecosistemas. En conclusión, y una vez clarificado el concepto de sustentabilidad, es posible establecer como su objetivo el mejoramiento, a largo plazo, de la calidad de vida del hombre, recurriendo al manejo e incluso transformación de la estructura y funcionamiento de los ecosistemas, pero teniendo presente el mantenimiento de los mismos.

\section{DIMENSIONES DE LA SUSTENTABILIDAD}

La sustentabilidad se proyecta a través de sus tres dimensiones, representadas esquemáticamente en la figura 3: medioambiental, progreso social y desarrollo económico.

La dimensión medioambiental o biofísica se refiere al mundo físico con el que y en el que, por ejemplo, la ganadería opera. Los atributos de esta dimensión pueden ser vistos como naturales, ya sean de naturaleza no renovable (minerales y combustibles), con un flujo predecible (aunque a veces también variable e incierto) como la luz del sol, la lluvia o el viento, o renovables como plantas, animales y en cierta medida el suelo y el agua (Yunlong y Smit, 1994). Es importante señalar, que la capacidad biológica de los recursos renovables de reponerse a sí mismos, si son tratados con el debido cuidado para salvaguardar las condiciones necesarias para su regeneración, se considera como la base de la sustentabilidad en esta dimensión. El equilibrio entre la energía que sale de un sistema agroganadero y la tasa de regeneración, natural, de la misma es clave (Massera et al., 1999 y Nahed et al., 2006). La introducción de energía de apoyo, combustibles, pesticidas, fertilizantes, piensos..., contribuye a deteriorar la sustentabilidad del sistema o de los sistemas externos que lo abastecen.

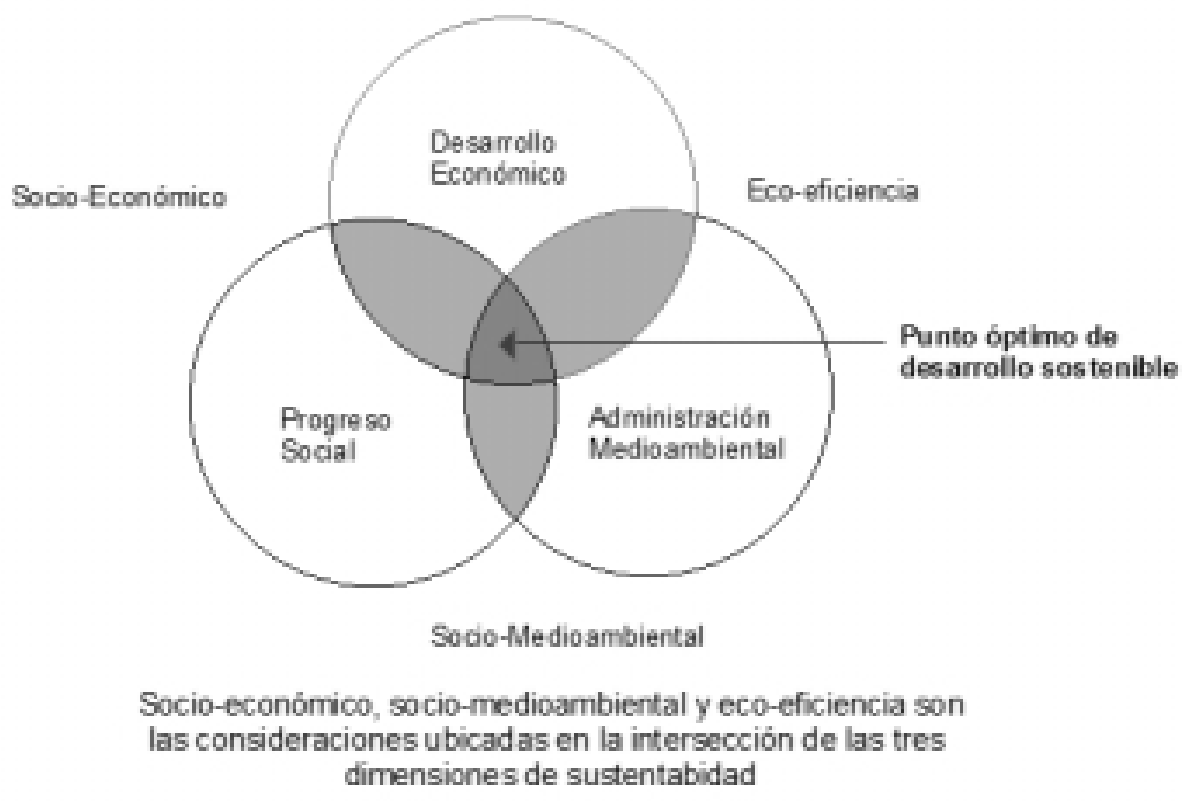

Figura 3. Dimensiones de la sustentabilidad. (Dimensions sustainability). 
El progreso social se relaciona con el papel que las poblaciones humanas, y las fuerzas culturales y sociales y otras fuentes de acción colectiva, desempeñan para influir en el comportamiento de las personas como individuos y como miembros de las familias, grupos y comunidades (Yunlong y Smit, 1994). Tres conceptos surgen como fundamentales dentro de esta dimensión, que están íntima y básicamente relacionados con los sistemas agropecuarios y giran en torno a ellos, estos son:

- La autosuficiencia o soberanía alimentaria, necesariamente basada en la agricultura y ganadería locales, que hace más independiente al productor tradicional de las oscilaciones del mercado, contribuyendo a su autonomía (La Via Campesina, 2003).

- La autonomía e independencia (procesos participativos, democráticos) que propician decisiones colectivas, estimulan y fortalecen la cultura local, las organizaciones que facilitan y protegen la producción y comercialización de sus productos, créditos y otros servicios, en condiciones justas y dan respaldo y potencia a la negociación con otras organizaciones y fuerzas sociales.

- Desarrollo endógeno y local (eje de desarrollo rural) que comprende el enriquecimiento cultural, la activación de mecanismos de autoestima e innovación, el desarrollo de mercados locales, que garanticen la estabilidad de los sistemas productivos y reduzcan la dependencia exterior de las comunidades, regiones e inclusive países.

La tercera dimensión, relacionada con el desarrollo económico y el ámbito tecnológico, actúa limitando en gran medida la viabilidad o factibilidad de las actividades productivas. Así en el caso de los agricultores o ganaderos, estos tienen que evaluar los costos asociados con diferentes combinaciones de insumos que pueden utilizarse en las actividades agropecuarias, la comercialización, los gastos de transpor- te e instalaciones y las cantidades de producto que se puede esperar con cada combinación (Fernandes and Woodhouse, 2008). Sin la probabilidad de obtener suficientes ganancias para cubrir por lo menos los costes de producción, los agricultores y ganaderos carecen de incentivos, y, en última instancia de capacidad, para el ejercicio de la agricultura o la ganadería. Los avances científicos y tecnológicos pueden tener gran impacto en la viabilidad económica y hacer viables las prácticas que no lo eran en anteriores circunstancias: cambios en los gastos de producción, en la comercialización, o en los precios de los productos (que reflejan la demanda de los consumidores también sometida a cambio), etc., pueden determinar, las circunstancias en las que la producción es económicamente viable (Yunlong y Smit, 1994).

Como se aprecia en la figura 3 , existen zonas de intersección entre las dimensiones. Para la obtención de una completa sustentabilidad debe haber una integración entre ellas (representando la triple intersección el punto óptimo del desarrollo sostenible). Las intersecciones entre dos dimensiones permiten considerar nuevos aspectos:

- Socio-económicos, como la creación de puestos de trabajo y otros impactos (positivos y negativos) de la relación entre la economía y el bienestar de la sociedad;

- Socio-medioambientales, que incluyen los efectos de la degradación de los recursos naturales (donde la participación de agricultura y ganadería es de especial relevancia) y también la liberación de emisiones al medio ambiente (donde tampoco están del todo exentas aquellas actividades del sector primario), la salud y la seguridad de las personas, para hoy y para las generaciones futuras (en lo que es obvia, asimismo, la responsabilidad de las producciones agrícola y ganadera);

- Eco-eficiencia, por ejemplo, la generación de un mayor valor (económico) al 


\section{SUSTENTABILIDAD DEAGROECOSISTEMAS}

usar menos recursos naturales y con un menor impacto medioambiental (Tanzil y Beloff, 2006), como sucede con la producción ganadera ecológica o en sistemas extensivos, donde el mayor valor económico deriva de la calidad superior obtenida y de las connotaciones éticas que enriquecen a los productos obtenidos en explotaciones respetuosas con el medio ambiente.

Wei et al. (2009) utilizó un modelo integrado para balancear las dimensiones económica, social y ambiental, en un sistema de cultivo de trigo y maíz con riego intensivo en la Llanura Norte de China, se representaron esquemáticamente los efectos del aumento del precio del agua y del nitrógeno en las tres dimensiones de la sustentabilidad. Para ello se utilizaron triángulos en los que cada vértice representa un nivel óptimo relativo de las dimensiones de la sustentabilidad, así, en la figura 4 (a), se aprecia como ante aumentos del precio del nitrógeno, la sustentabilidad económica disminuye, la sustentabilidad medioambiental aumenta y la social no se ve afectada. En el caso de un aumento en el precio del agua (figura 4 (b)) ocurre una situación diferente, dado que a medida que aumenta el costo de este insumo, disminuye la sustentabilidad económica, no afectándose las otras dos dimensiones de la sustentabilidad.

\section{Sustentabilidadecológica}

La definición de sustentabilidad ecológica se centra en los procesos biofísicos y la continuación de la productividad y el funcionamiento de los ecosistemas. A largo plazo la sustentabilidad ecológica exige el mantenimiento de una base de recursos de calidad, y eventualmente, su productividad, haciendo hincapié en el rendimiento sostenido de la tierra. La sustentabilidad ecológica exige también la preservación de las condiciones físicas, como son la hidrología superficial y subterránea, y el clima, además del mantenimiento de los recursos edáficos. Otras preocupaciones de orientación ecológica hacia la sustentabilidad son la protección de los recursos genéticos, tanto animales como vegetales y la conservación de la diversidad biológica (Yunlong y Smit, 1994).

La sustentabilidad ecológica encuentra su punto de referencia en la concepción
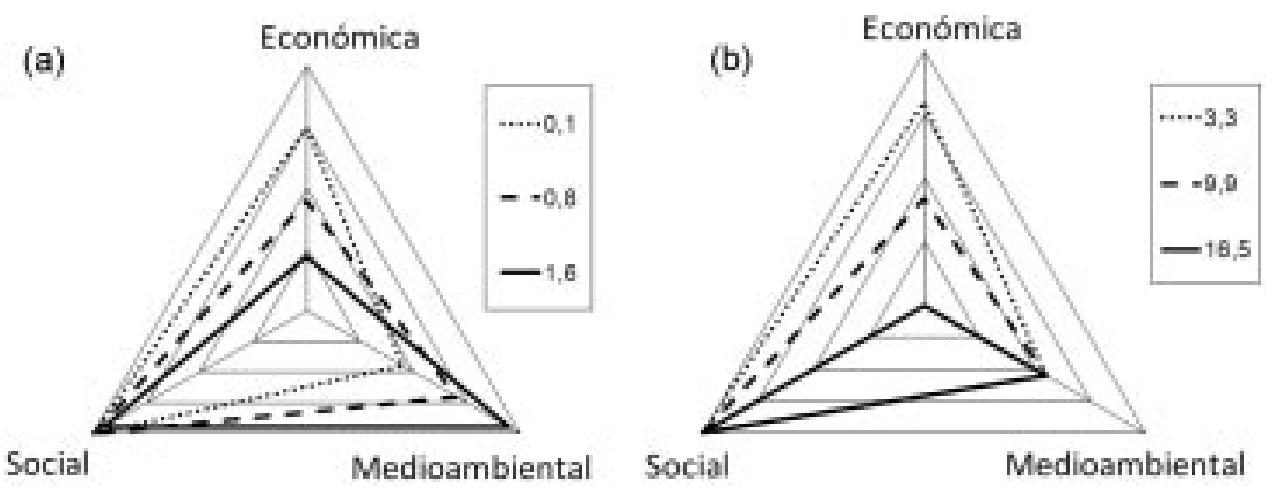

Adaptado de Wei et al. (2009).

Figura 4. Efecto del aumento de precio del nitrógeno (a) y agua (b) en las tres dimensiones de sustentabilidad en un agroecosistema. (Effect of increased price of nitrogen (a) and water (b) in the three dimensions of sustainability in an agroecosystem). 
de lo que se entiende por sociedad sostenible definida desde la perspectiva ecológica, como aquella (Allende, 2000) que:

- Conserva los sistemas ecológicos sustentadores de la vida y de la biodiversidad.

- Garantiza la sustentabilidad de recursos renovables y reduce a un mínimo el agotamiento de los recursos no renovables.

- Se mantiene dentro de la capacidad de carga de los ecosistemas sustentadores.

Un ejemplo notable de ello, lo constituye la dehesa, en la que el aprovechamiento ganadero secular ha demostrado la perfecta funcionalidad de los recursos naturales implicados: suelo-vegetaciónclima, de una lado, y producción ganadera, forestal, e incluso agrícola, de otro, en interacción armoniosa y protectora del medio, favoreciendo la diversidad biológica y contribuyendo a la conservación de un patrimonio genético (principalmente porcino, pero también de otras especies ganaderas) que ha sido depurado durante mucho tiempo por la selección, sin que el rendimiento se haya visto menoscabado (Rodríguez-Estévez et al., 2010).

\section{Sustentabilidad social}

La dimensión social de la sustentabilidad apunta a la continua satisfacción de las necesidades humanas básicas, la alimentación y la vivienda, así como a necesidades sociales y culturales de más alto nivel, como la seguridad, la equidad, la libertad, la educación, el empleo, y el ocio. Este concepto es definido por Barbier (1987) como la capacidad de mantener los valores sociales deseados, las tradiciones, las instituciones, las culturas y otras características sociales. En este sentido, la ganadería extensiva generadora de productos diferenciados por su calidad, y por tanto de mayor precio de venta, permite la consolidación en torno a ella de actividades rurales que, de este modo permiten la supervivencia de los pue- blos y la cultura asociada, al computar de forma integrada en la explotación todas las posibilidades productivas que genera el medio: obtención de productos de calidad, artesanía ligada a la actividad rural, agroturismo, etc. (García et al., 1999).

El concepto de sustentabilidad social, se introduce para pedir una solidaridad intrageneracional, en términos de justicia distributiva, que complemente la solidaridad intergeneracional que explícitamente se demanda en la formulación de desarrollo sostenible de la Comisión Mundial del Medio Ambiente y del Desarrollo (Comisión Mundial del Medio Ambiente y del Desarrollo, 1988) y que no se puede conseguir sin cambios en la política económica (Luffiego y Rabadán, 2000). En otras palabras, la solidaridad intrageneracional se refiere a la distribución justa y equitativa de los beneficios, a partir de la utilización de los recursos y la actividad agrícola entre los países, regiones o grupos sociales. En tanto que la intergeneracional, apunta a la protección de los derechos y oportunidades de las generaciones futuras para obtener beneficios de los recursos que hoy están en uso (Yunlong y Smit, 1994).

En el caso de los países en desarrollo, las demandas sociales imperativas son a menudo necesidades básicas del hogar o de la comunidad a corto plazo, a fin de evitar el hambre y las enfermedades (Yunlong y Smit, 1994). Sin embargo, en los países desarrollados, las demandas exigen proporcionar una cantidad y variedad de alimentos suficiente para satisfacer las demandas y preferencias actuales de los consumidores, además de garantizar un seguro suministro de alimentos (Smit y Smithers, 1994).

Los sistemas de producción agrícola o ganadera que contribuyen en alto grado al deterioro del medio ambiente no son considerados sostenibles, ya que a medida que pasen a las generaciones futuras aumentaran los costes de producción o los gastos de limpieza, que se añadirán a reducciones en 


\section{SUSTENTABILIDAD DEAGROECOSISTEMAS}

los ingresos o en la seguridad alimentaria, sin olvidar el deterioro general del medio ambiente derivado de estas actividades no sostenibles (por ejemplo emisiones de metano a la atmósfera). Los dos tipos de equidad a veces están relacionados aunque no siempre con el mismo signo; por ejemplo, muchos de los agricultores y ganaderos de subsistencia se ven obligados a emplear prácticas que proporcionan recompensas inmediatas, pero también degradan el medio ambiente y, por ende, perjudican las oportunidades para la sustentabilidad de las generaciones futuras (Barbier, 1987).

Refiriéndose a la importancia de la equidad entre generaciones Seifritz (2001) señala los siguientes hechos de relevancia:

- Los recursos no son el centro del universo en el marco de este tema, porque, lo que se denominan recursos, son sucesos dependientes del tiempo: llamamos a un cierto tipo de materia o a unas determinadas combinaciones de materiales un recurso, si en el mismo período de tiempo el conocimiento y la tecnología correspondiente están plenamente desarrollados y maduros para utilizar este recurso eficientemente. Por lo tanto, estos recursos son sucesos tiempo-dependientes generados a través de un progreso tecnológico.

- Dado que no se puede predecir la combinación de los recursos del futuro, no se sabe cuándo y que nuevas tecnologías tendrán éxito económico, ni se pueden basar las preguntas de sustentabilidad sobre la conservación y cuidado de cualquier tipo de material compuesto. Hay que decir claramente que la conservación de los recursos por sí sola conduce al posible bloqueo del surgimiento de nuevos conocimientos, es decir, una filosofía de la perplejidad.

- La única cosa que se sabe con certeza sobre el futuro es que, habrá que trabajar como ya ha teorizado Karl Marx, es decir, existirá una economía en la que el capital será el enlace entre todos los bienes y productos básicos. Por lo tanto, la principal tarea para todos, también para las genera- ciones futuras, es la preservación a largo plazo de la capacidad productiva de la economía. El ganador del premio Nobel de economía de 1987, Robert M. Solow expresó esto con las siguientes palabras: Si la sustentabilidad es algo más que un eslogan o una expresión de la emoción, debe equivaler a una orden judicial para preservar la capacidad productiva futura en forma indefinida (Solow, 1992).

Según Conway (1987) un claro objetivo de los agroecosistemas consiste en el aumento del valor social constituido por: la producción actual, su nivel probable en un horizonte temporal futuro y su distribución entre la población humana. Es decir, los bienes y servicios que producen los agroecosistemas en relación con las necesidades humanas y su distribución entre la población; considerando la dimensión temporal, esto es, no sólo los beneficios en el futuro inmediato, sino, también cierta seguridad a largo plazo. En este sentido deben considerarse, y asimilarse, las dificultades de implantación de los productos emergentes que, siendo más respetuosos con el medio ambiente y cumpliendo la función social de fijar las poblaciones alrededor de las explotaciones agrícolas y ganaderas, no tengan la recompensa inmediata de rentabilidad. Así ha sucedido y, en ciertos sectores de población, aun sigue sucediendo con la agricultura y ganadería ecológicas que han debido afrontar nuevas fórmulas productivas para abastecer a una población no preparada para entender todos los componentes del mayor precio de los productos que llevan su marchamo.

\section{Sustentabilidad económica}

La definición económica de la sustentabilidad se asocia principalmente a los beneficios percibidos a largo plazo por los productores. La sustentabilidad económica se inserta en el concepto de renta de Hicks, definida como la cantidad máxima que un individuo puede consumir en un período determinado de tiempo sin reducir su con- 
sumo en un período futuro (Hicks, 1945). En esta definición el cálculo de la renta, medido en términos de producto nacional o interior bruto, debe hacerse incluyendo la riqueza y los recursos medioambientales de un país, ya que en caso contrario, la medición no indicaría el grado de sustentabilidad.

Una versión de sustentabilidad económica apunta al rendimiento sostenido. En el caso particular de la agricultura y la ganadería, gran parte de la preocupación con respecto al rendimiento sostenido se deriva de los efectos de la degradación de las condiciones biofísicas de las tierras y el grado en que ello afecta a la productividad (Brklacich et al., 1991). Por supuesto, esta perspectiva se enreda con la definición ecológica de la sustentabilidad, aunque en este caso la preocupación se enfoca sobre la futura productividad y producción, en lugar de sobre los recursos naturales en sí (Yunlong y Smit, 1994).

En pocas palabras, sustentabilidad económica, significa evitar grandes perturbaciones y colapsos de cobertura, haciendo frente a la inestabilidad y discontinuidad (Costanza y Patten, 1995).

De este modo, actualmente la sustentabilidad de un sistema de producción depende de los retornos obtenidos, bajo un escenario con determinadas condiciones económicas y tecnológicas, una política socio-medioambiental definida, y una base de recursos naturales concretos. Es decir, en los sistemas contemporáneos la sustentabilidad no puede explicarse plenamente sin referencia a los tres conjuntos de condiciones. Además, para que un sistema sea sostenible debe ser posible biofísicamente, socio-políticamente aceptable, y técnica y económicamente viable (Yunlong y Smit, 1994).

En el caso de la actividad agraria Payraudeau y van der Werf (2005) declaran que desde el punto de vista medio-ambiental, la actividad agraria es sostenible si sus emisiones contaminantes y el uso de los recursos naturales pueden ser soportados a largo plazo por el medio ambiente natural. Desde el punto de vista económico, la sustentabilidad agraria abarca aspectos como la rentabilidad, la eficiencia y la productividad (James, 2006). En tanto que desde la perspectiva social los objetivos abarcan aspectos tales como el mantenimiento de familias de los agricultores y ganaderos, la búsqueda de alternativas que eviten el abandono de explotaciones de tamaño medio y la despoblación de comunidades rurales (Thompson, 1997). En esta dimensión de la sustentabilidad son considerados asimismo la mano de obra agraria y las condiciones de trabajo (Shreck et al., 2006).

En resumen, la sustentabilidad agroganadera se refiere a un sistema que integra salud medioambiental, rentabilidad económica y equidad social y económica. De modo tal, que la mayoría de las prácticas agrarias sostenibles y la gestión de la diversidad biológica sólo puede desarrollarse por medio de la comprensión del funcionamiento de los agroecosistemas, de la forma social, de la economía de agricultores y ganaderos $\mathrm{y}$ de sus entornos climáticos y edáficos $\mathrm{y}$ cómo éstos impactan sobre la productividad global de cultivos y animales (figura 5).

\section{VERSIONES DE SUSTENTABILIDAD}

El desarrollo del concepto de sustentabilidad, ha generado dos versiones del mismo: sustentabilidad débil y sustentabilidad fuerte (Norton, 1992). La sustentabilidad débil se ubica dentro del paradigma de la economía estándar, en tanto que la fuerte ha sido formulada por economistas heterodoxos, vinculados a la termodinámica y a la ecología (Naredo, 1996).

\section{Sustentabilidad débil}

La sustentabilidad débil es una concepción genérica que se define como la viabilidad de un sistema socioeconómico en el tiempo. Esta viabilidad se consigue manteniendo el capital global generación tras generación, siendo este capital global el resultado de otros dos: el capital natural y el capital humano (capital monetario, tecno- 
logía, personal formado, etc.). El capital natural es un concepto utilizado por los economistas para designar, por ejemplo el stock responsable del flujo de recursos naturales que entra en una sociedad, el bosque que produce un flujo de madera, el pasto que se transforma en una serie de productos animales (Luffiego, y Rabadán, 2000). En una versión más amplia del concepto Costanza y Patten (1992) incluyen los servicios y funciones básicos que presta la naturaleza a las sociedades humanas.

Desde el punto de vista de la sustentabilidad débil no existe incompatibilidad entre el crecimiento económico y la conservación del capital natural. Esta incompatibi- lidad se elude al admitir que los recursos que se agotan pueden ser sustituidos ilimitadamente siempre y cuando la tecnología evolucione (Mas-Colell, 1994). En el inicio de la sociedad humana el capital natural era inmenso, siendo el factor limitante del desarrollo el capital humano; sin embargo, actualmente el factor limitante comienza a ser el capital natural. La economía estándar sugiere la capacidad de sustitución de estos factores, por lo tanto no habría limitación alguna para el crecimiento (dogma central de la economía estándar). Esta visión reduccionista, sugiere que lo importante es la conservación de stock de capital global, no importando que el capital natural se de-

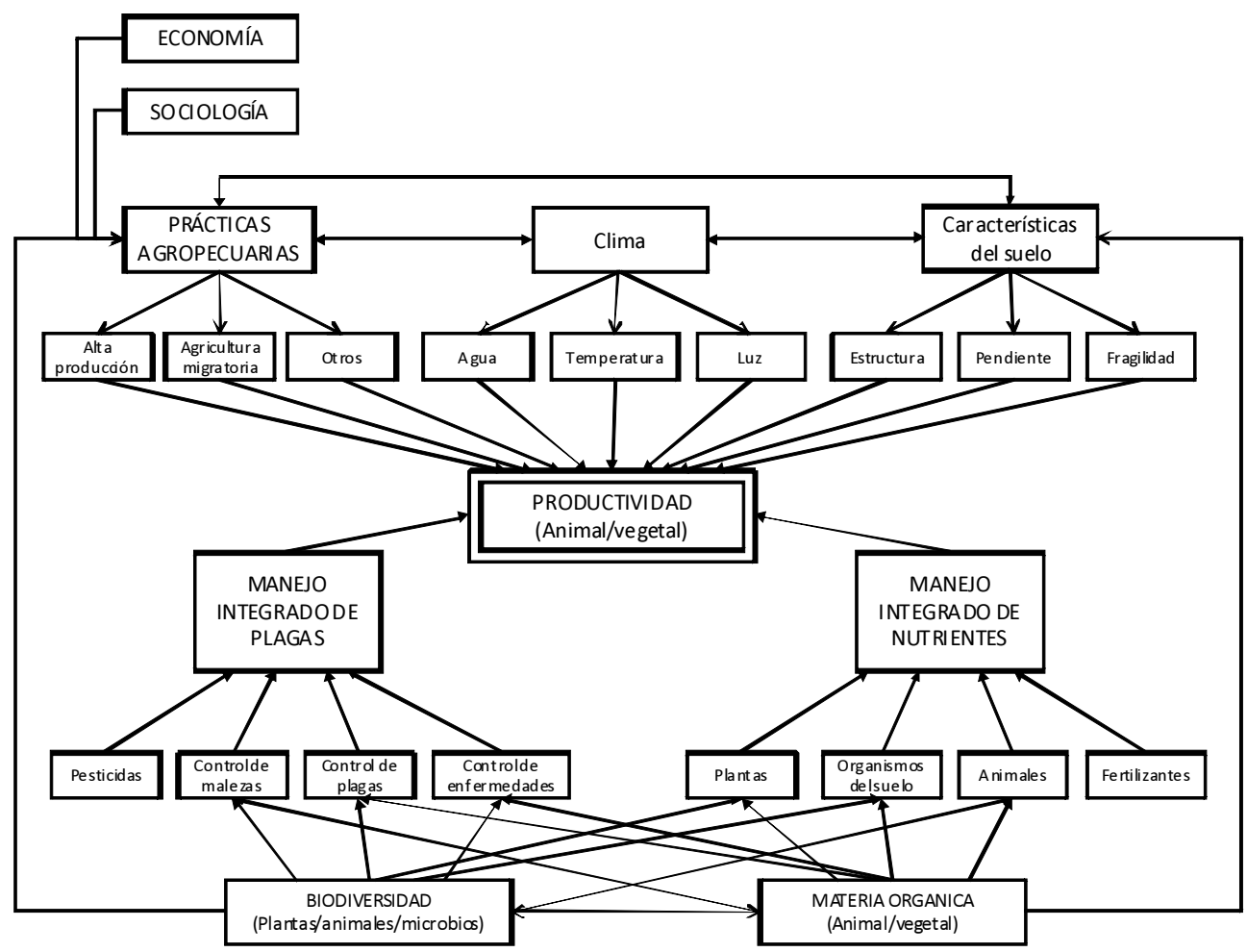

Adaptado de Edwards et al. (1993).

Figura 5. Procesos claves y relaciones funcionales en un agroecosistema sustentable. (Key processes and functional relationships in a sustainable agroecosystem). 
teriore, ya que una inversión siempre lo puede llegar a recuperar. Además, plantea la conveniencia para la salud medioambiental del crecimiento económico, dado que éste sería el mejor medio de recuperar la naturaleza deteriorada (Mas-Colell, 1994).

Resumiendo, la concepción de sustentabilidad débil se ubica en la órbita del paradigma mecanicista y reduccionista, propio de la economía actual, y reposa en una supeditación de la conservación de la naturaleza al crecimiento económico (Luffiego y Rabadán, 2000).

\section{Sustentabilidad fuerte}

Según Costanza y Patten (1992) en una breve y precisa definición la sustentabilidad fuerte es: la viabilidad de la interacción compleja entre dos sistemas dinámicos, el socioeconómico y el ecosistema, definición en la que también coincide Naredo (1994). Este concepto se basa en que la incertidumbre, la ignorancia y la irreversibilidad de los sistemas naturales, son de mayor entidad que los criterios de eficiencia económica (Jiménez Herrero, 2001).

El énfasis en esta definición es puesto en la interacción entre dos sistemas dinámicos, teniendo en cuenta que el sistema socioeconómico es dependiente del ecosistema en el sentido de que éste podría funcionar autónomamente, mientras que el socioeconómico no podría hacerlo sin el ecosistema. La interacción consiste, por lo tanto, en una permanente coadaptación (Luffiego y Rabadán, 2000).

La sustentabilidad fuerte, sería, por tanto la condición necesaria para la viabilidad en el tiempo de un sistema socioeconómico $\mathrm{y}$ al mismo tiempo impone una condición acerca de la naturaleza del sistema socioeconómico: ni el sistema económico ni el poblacional pueden mantener un crecimiento continuo.

Carpintero (1999) plantea utópicamente la necesidad de construir una economía ecológica, centrada en la utilización de los recursos locales y alimentada primordialmente por energía solar, lo que, sin embargo, no implicaría que esta economía sea cerrada, ya que los excedentes permitirían establecer intercambios entre regiones, para ajustar sus dimensiones a las del ecosistema del cual depende, por lo que habría no sólo un tipo de desarrollo sostenible sino una diversidad de evoluciones sostenibles.

A diferencia de la sustitución entre recursos planteada por la sustentabilidad débil, la sustentabilidad fuerte señala que el capital natural no es sustituible por ningún tipo de capital humano, siendo recursos complementarios (Daly, 1992). Pero, ¿qué es el capital natural?, según Ekins et al. (2003) y Pearce y Turner (1990), el capital natural posee cuatro categorías de funciones. En primer lugar, proporciona las materias primas para la producción y el consumo directo como alimentos, madera y combustibles fósiles. En segundo lugar, asimila los residuos de la producción y el consumo. En tercer lugar, ofrece comodidades y servicios, tales como la amenidad visual de un paisaje. En cuarto lugar, proporciona la base de sustentación de la vida de la que las funciones humanas, así como las tres primeras categorías de funciones del capital natural, dependen. De ahí que esta cuarta categoría no sólo sea un determinante directo del bienestar humano, sino que posee un valor primordial, al poder ser considerado un pegamento, que mantiene todo junto mientras que las tres primeras categorías son de un valor secundario (Turner et al., 1994).

Hoy en día, dada la existencia de una economía con presupuestos de crecimiento ilimitado, la sustentabilidad fuerte, puede ser considerada como un ideal utópico. Sin embargo, es posible comenzar a diseñar economías guiadas por principios derivados de la sustentabilidad fuerte y realizar proyectos concretos que, aunque enmarcados en la economía actual, se acerquen al ideal de sustentabilidad.

La sustentabilidad fuerte se identifica 


\section{SUSTENTABILIDAD DEAGROECOSISTEMAS}

con la sustentabilidad ecológica, ya que esta última asume el principio de complementariedad y subraya la incompatibilidad entre crecimiento y naturaleza (Luffiego y Rabadán, 2000).

Ejemplos que confirman la incapacidad de intercambio entre las distintas formas de capital, premisa de la sustentabilidad fuerte, son aquellos recursos naturales esenciales para el bienestar y/o supervivencia de la humanidad (conocidos como capital crítico), entre los que se puede mencionar la capa de ozono.

\section{DesarRollososteniBLE.}

Algunos expertos señalan que el concepto de tecnología apropiada, descrita por Schumacher (1989) y definida como la tecnología que toma en cuenta la habilidad, los niveles de la población, la disponibilidad de los recursos naturales y las necesidades sociales apremiantes, es el precursor inmediato del concepto de desarrollo sostenible (Mebratu, 1998). Así, el concepto de desarrollo sostenible surge al final de los años sesenta, como resultado de la consciencia de los crecientes problemas ambientales y de los límites impuestos por la naturaleza a la explotación y crecimiento económico descontrolado con la pretensión de integrar y asociar las dimensiones económica y social con la dimensión ecológica (Cardoso y Faletto, 1975).

Aunque se han propuesto numerosas definiciones de desarrollo sostenible, en general, son similares a la de la Comisión Mundial sobre Medio Ambiente y el Desarrollo de 1988: desarrollo sostenible es el que satisface las necesidades del presente sin comprometer la capacidad de las generaciones futuras para satisfacer sus propias necesidades.

En la definición conceptual de la Comisión Brundtland (Comisión Mundial del Medio Ambiente y del Desarrollo, 1988), se encuentran dos conceptos claves:

- El concepto de necesidades, en particular las necesidades esenciales de los po- bres, a las que se debe dar la mayor prioridad.

- La idea de las limitaciones impuestas por el estado de la tecnología y la organización social sobre la capacidad del medioambiente para satisfacer las necesidades del presente y del futuro.

Al hacerlo, la Comisión subraya el fuerte vínculo entre el alivio de la pobreza, la mejora del medioambiente y la equidad social a través de un crecimiento económico sostenible. De este modo, el significado de desarrollo sostenible parece ser el de un desarrollo viable en el tiempo cuya condición esencial es que las capacidades del sistema socioeconómico no disminuyan y puedan estar a disposición de las generaciones venideras (Luffiego y Rabadán, 2000). Sin embargo, no se debe olvidar que el sistema socioeconómico es netamente dependiente del medio natural y por lo tanto, este debe ser el bien primordialmente a proteger.

Neumayer (2003), señala que una regla intergeneracional aparentemente simple es que se está frente a un desarrollo sostenible si no se disminuye la capacidad de ofrecer una utilidad per cápita dada hasta el infinito. Sharachchandra (1991), desde una revisión crítica del concepto de desarrollo sostenible, señala que existen distintas interpretaciones del término, pero en la utilizada por la mayoría de las personas, se entiende como intercambiable por ecológicamente sostenible o buen desarrollo medioambiental, mencionando que ésta interpretación se caracteriza por:

(a) la sustentabilidad debe ser entendida como la sustentabilidad ecológica.

(b) el desarrollo sostenible es un proceso de cambio que añade la sustentabilidad (ecológica) a su lista de objetivos.

Pero también, el desarrollo sostenible es a veces interpretado como un crecimiento sostenido, un cambio sostenido, o simplemente un desarrollo exitoso.

La capacidad de proporcionar utilidad 
es conceptualmente atribuida en cuatro formas de capital: producción, recursos naturales, humanos y sociales. Para hacer frente a las actuales necesidades, la economía tiene por objeto abordar la escasez de suministro. Las dimensiones de la escasez y de la oferta son un resultado de la heterogeneidad espacial de los niveles local a global. Satisfacer las necesidades de las generaciones futuras requiere el reconocimiento de la heterogeneidad temporal a corto y largo plazo. Por otra parte, la dimensión temporal de los recursos corresponde a la incorporación de la variación natural y sostenible en los ciclos de los sistemas económicos. Este ciclo de tiempo limita la asignación de recursos de tal forma que se clasifica como norenovables a los que presentan una larga tasa de regeneración, o tienen una velocidad de regeneración inferior a la tasa de consumo y como renovables a aquellos con cortas tasas de regeneración (Allen y Hoekstra, 1992).

El desarrollo sostenible se ha convertido en uno de los tópicos mundiales más populares de la agenda política de casi todos los gobiernos, que declaran comprometerse en este empeño mediante la integración del bienestar económico, la calidad ambiental y la coherencia social (Böhringer y Jochem, 2007).

Se observan posturas enfrentadas entre el concepto desarrollo y medioambiente. Según Vilches y Gil (2003), el concepto de desarrollo sostenible no propone ver al desarrollo y al medioambiente como contradictorios (el primero agrediendo al segundo y éste limitando al primero) sino que reconoce que están estrechamente vinculados, no pudiendo ser tratados por separado la economía y el medioambiente. Se estaría produciendo una revolución en la que se integre ambiente y desarrollo, mediante un moderno y confuso paradigma que pretende ensamblar en íntima simbiosis la gestión ambiental y el desarrollo económico (Allende, 2000). Por otro lado, diversos autores señalan una relación competitiva entre desa- rrollo y medioambiente dónde el binomio desarrollo sostenible evidencia una contradicción en su propio nombre. Tal afirmación la justifican en el origen de los términos: desarrollo que ha sido tomado desde la economía de mercado y sostenibilidad, proveniente desde las ciencias ecológicas y biológicas (Boff, 2006). Desde el campo de la educación científica, la ambigüedad del concepto, es atribuida a que, al mismo tiempo que se acepta la existencia de límites a los modos de vida que no sean compatibles con los principios ecológicos, se mantiene la creencia en el crecimiento o desarrollo para satisfacer las necesidades humanas (Luffiego y Rabadán, 2000).

La admisión de la asociación existente en el término desarrollo sostenible, parte de la suposición de que puede haber desarro1lo, mejora cualitativa o despliegue de potencialidades, sin crecimiento, es decir, sin incremento cuantitativo de la escala física, sin incorporación de mayor cantidad de energía ni de materiales. En otras palabras, es el crecimiento lo que no puede continuar indefinidamente en un mundo finito, pero sí es posible el desarrollo (Macedo, 2005).

Las consideraciones ecológicas sugieren que los niveles y grados de la actividad económica actual no son sustentables, situación generada por la inherente tendencia de la actividad humana de simplificar ecosistemas, reducir su actividad biológica (por ejemplo monocultivo agrícola, explotaciones intensivas de especies ganaderas especializadas en una determinada producción) y consecuente reducción de su estabilidad (Cosio, 1999). De este modo, se reconoce que los beneficios del crecimiento económico no se pueden lograr a largo plazo, a menos que la forma de desarrollo tenga en cuenta su impacto ambiental. Existen numerosos ejemplos de las intervenciones del gobierno en el mercado, contrarios al concepto de la sustentabilidad y la protección del medio ambiente tanto en países desarrollados como en vías de desarrollo. 


\section{SUSTENTABILIDADDE AGROECOSISTEMAS}

Tabla I. Componentes del desarrollo rural sustentable. (Components of sustainable rural development).

\begin{tabular}{llll}
\hline Calidad de vida & Desarrollo humano & Desarrollo agrícola & Apoyo externo \\
\hline Salud & Educación & Manejo de los factores de producción & Crédito \\
Alimentación & Formación & Adecuada producción y calidad & Seguros \\
Vivienda & Administración & & Ahorro \\
Vestuario & Negociación & & Subsidios \\
Servicios & Liderazgo & & Asesoría \\
\hline
\end{tabular}

Un ejemplo de estas intervenciones es la entrega continua de subvenciones al sector agrícola y ganadero en Europa, para drenar y mejorar las áreas naturales (Bidwell, 1992).

En el término desarrollo sostenible además de la ecología y la economía confluyen otras disciplinas, como la ética, la política, la sociología, el derecho o la cultura, como expone Font (2000): Las diferentes disciplinas que se han ocupado de conceptualizar el desarrollo sostenible se interesan por cuestiones de diversa indole: la perspectiva ecológica pone el acento en la necesidad de mantener la biodiversidad y los ecosistemas; la economía se interesa, entre otras cuestiones por la internalización de las externalidades; el discurso ético recurre a principios filosóficos universales, y la ciencia política se preocupa por la gobernabilidad y la justicia distributiva.

En el mismo ámbito, la producción agropecuaria no puede desvincularse de su papel dentro del desarrollo sostenible, dado que a través de sus tecnologías y prácticas de cría y cultivo condiciona el grado de adaptabilidad y perpetuidad de sistemas originalmente naturales a su nuevo entorno productivo. La agricultura sostenible, mencionada en párrafos anteriores, no es más que el concepto de desarrollo sostenible enfocado en agricultura. En la tabla I, se muestran los componentes que Bustillo et al. (2008) utilizan como ayuda en la búsqueda de un desarrollo igualitario, sustentable y sostenido en una propuesta de desarrollo rural.

\section{CONCLUSIONES}

La sustentabilidad de los agroecosistemas depende de sus características básicas y de cómo, por qué, y a través de que variables éstas son afectadas dentro de cada dimensión. Para establecer la resiliencia de un agroecosistema deben valorarse la estabilidad económica, ecológica y social, especialmente en lo que se refiere a la productividad, la rentabilidad económica de la actividad y la creación de empleo endógeno.

La conveniencia en el análisis de los agroecosistemas no debe considerarse desde una óptica antropocéntrica sino de un modo más amplio (holístico) que favorezca la sustentabilidad de los sistemas de producción.

La sustentabilidad débil debe ser abandonada como opción, dada la imposibilidad de sustitución completa entre capital natural y humano. Los indicadores de biodiversidad, calidad del suelo, agua y aire, que permitan evaluar la condición del agroecosistema para el conjunto natural de organismos vivos cobran especial importancia.

La escala, en su variación tanto espacial como temporal, permite una cierta adaptación conveniente a la situación o sistema analizado; teniendo en cuenta la jerarquía y complejidad de los agroecosistemas pecuarios. Para fijar los límites espaciales y temporales de un agroecosistema, se necesita conocer y evaluar, junto con los ciclos de producción de las especies comerciales, los 
nichos ecológicos y sus interacciones.

La utilización del predio como unidad de estudio, resulta satisfactoria en la medida que se consideren y evalúen las interacciones de las distintas actividades realizadas, junto con las externalidades y la complementación e interferencia con las actividades de predios adyacentes.

Sistemas de producción sustentables, donde la versión de sustentabilidad predominante es la fuerte, comparten gran parte de sus principios con la producción eco-

\section{BIBLIOGRAFÍA}

Allen, T. and Hoekstra, T. 1992. Toward a unified ecology. Columbia University Press. New York. $384 \mathrm{pp}$.

Allende, J. 2000. Medio ambiente, ordenación del territorio y sostenibilidad. Bilbao. Servicio Editorial. Universidad del País Vasco. 274 pp.

Altieri, M.A. 1989. Agroecology: A new research and development paradigm for world agriculture. Agr. Ecosyst. Environ., 27: 37-46.

Altieri, M.A. 1992a. Agroecological foundations of alternative agriculture in California. Agric., Ecosyst. Environm., 39: 23-53.

Altieri, M.A. 1992b. Sustainable agricultural development in Latin America: exploring the possibilities. Agr. Ecosyst. Environm., 39: 1-21.

Barbier, E. 1987. The concept of sustainable economic development. Environ. Conserv., 14: 101-110.

Bidwell, R. 1992. Sustainability: The link between conservation and economic benefits. Environm. Impact Assess. Rev., 12: 37-47.

Boff, L. 2006. La contradicción capitalismo/ ecología. Ecoportal.net. El directorio ecológico y natural. www.ecoportal.net. (28/07/06).

Böhringer, C. and Jochem, P. 2007. Measuring the immeasurable. A survey of sustainability indices. Ecol. Econ., 63: 1-8.

Brklacich, M., Bryant, C. and Smit, B. 1991. Review and appraisal of concept of sustainable food production system. Environm. Manage., 15: 1-14.

Bustillo, L., Martínez, J. y Gallardo, F. 2008. El desarrollo rural sustentable: ese ilustre desconocido (una revisión). Rev. Cient. FCV-LUZ, 18: $43-50$. lógica, entre los que destacan la integración de procesos biológicos y ecológicos, la minimización del uso de insumos no renovables, la utilización de razas autóctonas y la equidad tanto medioambiental como social.

\section{AGRADECIMIENTOS}

La autora principal agradece a la Agencia Española de Cooperación Internacional por el aporte financiero recibido durante la realización de sus estudios de Postgrado.

Campos, P. y Carrera, M. 2007. Crítica de la exclusión del aprovechamiento de recursos naturales en los parques nacionales españoles. Principios: Estudios de Economía Política, 8: 39-58.

Cardoso, F. e Faletto, E. 1975. Dependência e desenvolvimento na América Latina. Zahar Editores. Rio de Janeiro.

Carpenter, S., Walker, B., Anderies, M. and Abel, N. 2001. Minireview. From metaphor to measurement: Resilience of what to what? Ecosystems, 4: 765-781.

Carpintero, O. 1999. Entre la economía y la naturaleza. Fundación $1^{\circ}$ de Mayo. Madrid. 382 pp.

Castel, J., Mena, Y., Delgado-Pertinez, M., Camunez, J., Basulto, J., Caravaca, F., GuzmanGuerrero, J. and Alcalde, M. 2003. Characterization of semi-extensive goat production systems in southern Spain. Small Rum. Res., 47: 133-143.

Catrileo, A., Toro, P., Aguilar, C. and Vera, R. 2009. Use of supplements and variation in the stocking rate in cow-calf systems on temperate pastures in Chile: A simulation approach. Anim. Prod. Sci., 49: 1059-1067.

Comisión Mundial del Medio Ambiente y del Desarrollo, 1988. Nuestro futuro común. Alianza Editorial S.A. Madrid. 460 pp.

Conway, G.R. 1987. The properties of agroecosystems. Agric. Syst., 24: 95-117.

Cosio, F. 1999. Determinación y análisis de la estructura técnico-económica para la asignación de derechos de uso de pastizales en la veranada de montaña. Laguna del Maule. Chile. 


\section{SUSTENTABILIDAD DEAGROECOSISTEMAS}

Departamento de Producción Animal. Universidad de Córdoba. Córdoba. España. 285 pp.

Costanza, R. y Patten, B. 1992. La economía ecológica de la sostenibilidad. Invertir en capital natural. En: Goodland, R. et al. (Eds.). Medio ambiente y desarrollo sostenible. Trotta. Madrid. pp: 103-114.

Costanza, R. and Patten, B. 1995. Commentary: Defining and predicting sustainability. Ecol. Econ., 15: 193-196.

Daly, H. 1992. De la economía del mundo lleno a la economía del mundo vacío. En: Goodland, R. et al. (Eds.). Medio ambiente y desarrollo sostenible. Trotta. Madrid. pp. 35-50.

Edwards, C., Grove., T., Harwood, R. and Colfer, P. 1993. The role of agroecology and integrated farming systems in agricultural sustainability. Agr. Ecosyst. Environ., 46: 99-121.

Ekins, P., Simon, S., Deutsch, L., Folke, C. and de Groot, R. 2003. A framework for the practical application of the concepts of critical natural capital and strong sustainability. Ecol. Econ., 44: 165-185.

Fernandes, L. and Woodhouse, P.J. 2008. Family farm sustainability in southern Brazil: An application of agri-environmental indicators. Ecol. Econ., 66: 243-257.

Fernández, G. 2005. Alternativas de gestión con ganadería bovina en sistemas pastoriles de la Región Semiárida Pampera (Argentina). Sistema de cría y engorde de la propia producción. Departamento de Producción Animal. Universidad de Córdoba. Córdoba. España. 277 pp.

Fiksel, J., 2006. Sustainability and resilience: toward a systems approach. Sustainability: Science, Practice, and Policy, 2: 14-21.

Folch, R. 1998. Ambiente, emoción y ética. Ed. Ariel. Barcelona. 191 pp.

Font, N. 2000. Local y sostenible. En: Font, N. y Subirats, J. (Eds.). Local y sostenible: la agenda 21 local en España. Ed. Editorial Icaria. Barcelona. pp. 9-28.

Frías, J. 1998. Situación actual y perspectivas de conservación de las razas caprinas en peligro de extinción en la Provincia de Jaén. Producción Animal. Universidad de Córdoba. Córdoba. España.

García, P., Díaz, F., Sánchez, M. and García, F. 2009. Assessment of the sustainability in dehesa farms according to size and livestock prevalence. ITEA, 105: 117-141.

García, A., Domenech, V., Frías, J., Herrera, M., Peña, F., Martos, J. y Acero, R. 1999. Caracterización técnico-económica de los sistemas de producción caprino extensivo en la provincia de Jaén como base del desarrollo sostenible. Analistas Económicos de Andalucía. Sevilla. $124 \mathrm{pp}$.

Giorgis, A. 2009. Factores que afectan la competitividad de las empresas agropecuarias de la zona norte de la Provincia de la Pampa. Argentina. Universidad de Córdoba. Córdoba. España. 220 pp.

Gliessman, S. 1998. Agroecology: Ecological process in sustainable agriculture. Ann Arbor Press. Michigan.

Gliessman, S. 1995. Sustainable agriculture: An agroecological perspective, In: J.H. Andrews and I.C. Tommerup (Eds.). Advances in Plant Pathology. Academic Press. New York. 11: 4557.

Grimm, V. and Wissel, C. 1997. Babel, or the ecological stability discussions: An inventory and analysis of terminology and a guide for avoiding confusion. Oecologia, 109: 323-334.

Gunderson L. and Holling C. 2001. Panarchy: understanding transformations in human and natural systems. Island Press. Washington, DC. $507 \mathrm{pp}$.

Halberg, N. 1999. Indicators of resource use and environmental impact for use in a decision aid for Danish livestock farmers. Agr. Ecosyst. Environ., 76: 17-30.

Heitschmidt, R.K., Short, R.E. and Grings, E.E. 1996. Ecosystems, sustainability, and animal agriculture. J. Anim. Sci., 74: 1395-1405.

Hicks, J.R. 1945. Valor y capital. Investigación sobre algunos principios fundamentales de teoría económica. Fondo de Cultura Económica. México. $336 \mathrm{pp}$.

IFOAM. 2005. Principles of Organic Agriculture, IFOAM General assembly 2005. Adelaide. Disponible en: http://www.ifoam.org/about_ifo2am/ pdfs/POA_folder_spanish.pdf. (01/12/10).

James, H.S. 2006. Sustainable agriculture and free market economics: Finding common ground in Adam Smith. Agricult. Hum. Values, 23: 427438.

Jiménez-Herrero, L. 2001. Desarrollo sostenible y economía ecológica. Integración medio ambien- 


\section{TORO-MÚJICA, GARCÍA, GÓMEZ-CASTRO, ACERO, PEREA YRODRÍGUEZ-ESTÉVEZ}

te, desarrollo y economía-ecología. Ediciones Síntesis. Madrid. 366 pp.

Labandeira, X., León, C. y Vázquez, M. 2007. Economía ambiental. Pearson Educación, S.A. Madrid. $376 \mathrm{pp}$.

La vía campesina. 2003. Posición sobre soberanía alimentaria de los pueblos. Disponible en: http://viacampesina.org/sp/index.php?option= com_content\&view=article\&id=345:posicionsobre-soberania-alimentaria-de-los-pueblos \&catid $=21$ : soberanalimentary-comercio\& Itemid=38. (01/12/10).

López, V. 2001. Sustainable development. A conceptual and operative approach to sustainability principles for the construction sector. Universitat Politécnica de Catalunya. 235 pp.

Luffiego, M. y Rabadán, J. 2000. La evolución del concepto de sostenibilidad y su introducción en la enseñanza. Enseñanza Ciencias, 18: 473-486.

Macedo, B. 2005. El concepto de sostenibilidad. Organización de las Naciones Unidas para la Educación, la Ciencia y la Cultura. Oficina Regional de Educación para América Latina y el Caribe UNESCO Santiago. Disponible en: http:// unesdoc.unesco.org/images/0016/001621/ 162177S.pdf. (01/10/08).

Marquardt, B. 2006. Historia de la sostenibilidad. Un concepto medioambiental en la historia de Europa central (1000-2006). Historia Crítica, 32: 172-197.

Marten, G.G. 1988. Productivity, Stability, Sustainability, equitability and autonomy as properties for agroecosystem assessment. Agric. Syst., 26: 291-316.

Mas-Colell, A. 1994. Elogio del crecimiento económico. En: Nadal, J. (Ed.). El mundo que viene. Alianza. Madrid. pp. 189-220.

Masera, O., Astier, M. y López-Ridaura, S. 1999. Sustentabilidad y manejo de recursos naturales. El marco de evaluación MESMIS. Mundi-Prensa, S.A. Gira. IE-UNAM. México. 109 pp.

Mayer, A. 2008. Strengths and weaknesses of common sustainability indices for multidimensional systems. Environm. Int., 34: 277291.

Mebratu, D. 1998. Sustainability and sustainable development: Historical and conceptual review. Environm. Impact Assess. Rev., 18: 493-520. Meul, M., Van Passel, S., Nevens, F., Dessein, J.,
Rogge, E., Mulier, A. and Van Hauwermeiren, A. 2008. MOTIFS: a monitoring tool for integrated farm sustainability. Agron. Sustain. Dev., 28: 321-332.

Morán, M. 2000. Desarrollo de las medidas agroambientales- en el marco de la Política Agraria Comunitaria (PAC II-III). An. Geogr. Univ. Complutense, 20:349-363.

Nahed, J., Castel, J.M., Mena, Y. and Caravaca, F. 2006. Appraisal of the sustainability of dairy goat systems in Southern Spain according to their degree of intensification. Livest. Sci., 101:10-23.

Naredo, J. 1994. Fundamentos de la economía ecológica. En: Aguilera, F. y Alcántara, V. (Eds.). De la economía ambiental a la economía ecológica. Icaria. Barcelona. pp. 373-404.

Naredo, J. 1996. Sobre el origen, el uso y el contenido del término sostenible. Doc. Social, 102: 129-147.

Neumayer, E. 2003. Weak versus strong sustainability: Exploring the limits of two opposing paradigms. Edward Elgar. Northampton, MA. $271 \mathrm{pp}$.

Norton, B, 1992. Sustainability, human welfare and ecosystem health. Ecol. Econ., 14: 113-127.

Okey, B.W. 1996. Systems approaches and properties, and agroecosystem health. J. Environm. Manage., 48: 187-199.

Parris, T. and Kates, R. 2003. Characterizing and measuring sustainable development. Ann. Rev. Environm. Resourc., 28: 13-31.

Payraudeau, S. and Van der Werf, H.M.G. 2005. Environmental impact assessment for a farming region: a review of methods. Agr. Ecosyst. Environ., 107: 1-19.

Pearce, D. and Turner, R. 1990. Economics of natural resources and the environment. Harvester Wheatsheaf. Hemel Hempstead. 378 pp.

Pretty, J. 2008. Agricultural sustainability: concepts, principles and evidence. Philosoph. Trans. Roy. Soc. B-Biol. Sci., 363: 447-465.

Rigby, D. and Cáceres, D. 2001. Organic farming and the sustainability of agricultural systems. Agricult. Syst., 68: 21-40.

Rodríguez-Estévez, V., Sánchez-Rodríguez, M., Gómez-Castro, A.G. and Edwards, S.A. 2010. Group sizes and resting locations of free range pigs when grazing in a natural environment. Appl. Anim. Behav. Sci., 127: 28-36.

Schaller, N. 1993. Sustainable agriculture and the 


\section{SUSTENTABILIDAD DEAGROECOSISTEMAS}

environment. The concept of agricultural sustainability. Agr. Ecosyst. Environm., 46: 8997.

Schumacher, E. 1989. Small is beautiful. Harper Perennial. London. $286 \mathrm{pp}$.

Seifritz, W. 2001. On the eclectic concept of sustainability. Int. J. Hydrogen Energy, 26: 1017-1022.

Sharachchandra, M. 1991. Sustainable development: A critical review. World Develop., 19: 607-621.

Shreck, A., Getz, C. and Feenstra, G. 2006. Social sustainability, farm labor, and organic agriculture: Findings from an exploratory analysis. Agricult. Hum. Values, 23: 439-449.

Smit, B. and Smithers, J. 1994. Sustainable agriculture: Interpretations, analyses and prospects. Can. J. Reg. Sci., 16: 499-524.

Solow, R. 1993. An almost practical step toward sustainability. Resources Policy, 19: 162-172.

Speeding, C.R.W. 1995. Sustainability in animal production systems. Anim. Sci., 61: 1-8.

Tanzil, D. and Beloff, B. 2006. Assessing Impacts: Overview on sustainability indicators and metrics. Environm. Qual. Manage., 15: 41-56.

Thompson, P. 1997. Sustainability as a norm. Soc. Philosophy Technol., 2: 75-93.

Toro, P., Catrileo, A., Aguilar, C. and Vera, R. 2009. Modelling supplementation strategies for beef steer rearing and fattening systems in southern Chile. Chilean J. Agric. Res., 69: 207-213.

Toro-Mujica, P., García, A., Gómez-Castro, A.G., Acero, R., Perea, J., y Rodríguez-Estévez, V., 2010. Sustentabilidad en agroecosistemas. Arch. Zootec., 59: 71-94.

Troell, M., Pihl, L., Rönnbäck, P., Wennhage, H., Söderqvist, T. and Kautsky, N. 2005. Regime shifts and ecosystem services in Swedish coastal soft bottom habitats: when resilience is undesirable. Ecol. Soc., 10: 30.

Turner, R., Pearce, D. and Bateman, I. 1994. Envi- ronmental economics: An elementary introduction. Harvester. Hemel Hempstead. 328 pp.

Ulgiati, S. and Brown, M. 1998. Monitoring patterns of sustainability in natural and manmade ecosystems. Ecol. Model., 108: 23-36.

Van Ittersum, M.K. and Rabbinge, R. 1997. Concepts in production ecology for analysis and quantification of agricultural input-output combinations. Field Crops Res., 52: 197-208.

Valerio, D., García, A., Perea, J., Acero, R. y Gómez, G. 2009. Caracterización social y comercial de los sistemas ovinos y caprinos de la región noroeste de República Dominicana. Interciencia, 34:637-644.

Vavra, M. 1996. Sustainability of animal production systems: An ecological perspective. J. Anim. Sci., 74: 1418-1423.

Viglizzo, E.F. 1986. Agroecosystems stability in the Argentine Pampas. Agr. Ecosyst. Environ., 16: 1-12.

Viglizzo, E.F. and Roberto, Z.E. 1998. On trade-offs in low-input agroecosystems. Agric. Syst., 56: 253-264.

Vilches, A. y Gil, D. 2003. Construyamos un futuro sostenible. Diálogos de supervivencia. Cambridge University Press. Madrid. 275 pp.

Wei, Y., Davidson, B., Chen, D. and White, R. 2009. Balancing the economic, social and environmental dimensions of agro-ecosystems: An integrated modeling approach. Agr. Ecosyst. Environ., 131: 263-273.

Weil, R. 1990. Defining and using the concept of sustainable agriculture. J. Agron. Ed., 19: 126130.

Youngberg, G. and Harwood, R. 1989. Sustainable farming systems: Needs and opportunities. Amer. J. Alt. Agric., 4: 100.

Yunlong, C. and Smit, B. 1994. Sustainability in agriculture: A general review. Agr. Ecosyst. Environ., 49: 299-307. 